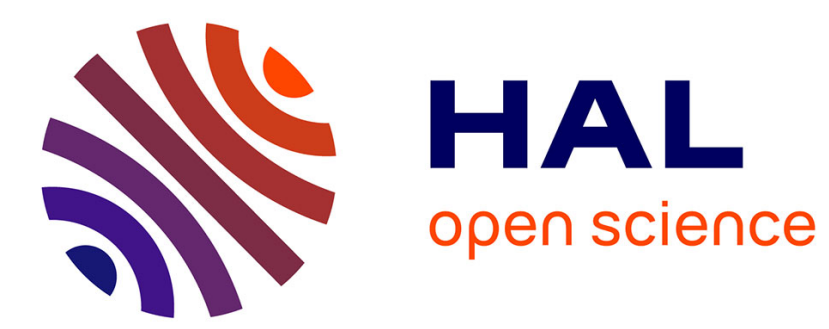

\title{
NON-LINEAR FREE PERIODIC OSCILLATIONS OF A TETHERED SATELLITE SYSTEM
}

\author{
Angelo Luongo, Fabrizio Vestroni
}

\section{To cite this version:}

Angelo Luongo, Fabrizio Vestroni. NON-LINEAR FREE PERIODIC OSCILLATIONS OF A TETHERED SATELLITE SYSTEM. Journal of Sound and Vibration, 1994, 175 (3), pp.299-315. hal00812015

\section{HAL Id: hal-00812015 https://hal.science/hal-00812015}

Submitted on 11 Apr 2013

HAL is a multi-disciplinary open access archive for the deposit and dissemination of scientific research documents, whether they are published or not. The documents may come from teaching and research institutions in France or abroad, or from public or private research centers.
L'archive ouverte pluridisciplinaire HAL, est destinée au dépôt et à la diffusion de documents scientifiques de niveau recherche, publiés ou non, émanant des établissements d'enseignement et de recherche français ou étrangers, des laboratoires publics ou privés. 


\title{
NON-LINEAR FREE PERIODIC OSCILLATIONS \\ OF A TETHERED SATELLITE SYSTEM
}

\author{
A. LUONGO AND F. VESTRONI \\ Dipartimento di Ingegneria delle Strulture, Acque e Terreno, Università dell'Aquila, \\ Monteluco di Roio, 67040 L'Aquila, Italy
}

This paper-deals with the non-linear dynamics of a tethered satellite system, with particular reference to periodic transverse oscillations. The motion is governed by two integro-differential equations with quadratic non-linear terms associated to gyroscopic forces. Notwithstanding the weakness of the non-linearities, the closeness of the in-plane and out-of-plane frequencies suggest that it would be interesting to study the periodic finite oscillations and analyze their stability. Since the system operates virtually in conditions of internal resonance, a certain number of modes are involved in the phenomenon. An asymptotic analysis of the partial differential equations of the continuous system makes it possible to take into account the fact that both the frequencies and the oscillation shapes are amplitude-dependent. Primary und secondary instability phenomena are investigated by using the Floquet theory and approximate analytical expressions of the unstable regions are obtained.

\section{INTRODUCTION}

Since the proposal to run a series of experiments in space with a satellite tethered to an orbiting station first appeared [1-3], much ground has been covered and substantial research has been developed. Considerable efforts have been dedicated to the study of the dynamic behaviour of the tethered satellite system (TSS) both during deployment and retrieval of the satellite and during the station-keeping phase [4-6]. On this last aspect, most papers have been limited in scope mainly to linear dynamics, while outlining some important specific points [7-11]. The dynamical behaviour of the system is such that the longitudinal and transversal oscillations are coupled by gyroscopic forces, but the coupling is so weak that two distinct sets of eigenfunctions exist, one described by the prevailing longitudinal displacement component and the other by the transverse component $[8,9]$.

By focusing on the transverse oscillations, which are more easily excited by various sources of disturbance, it has been shown that the frequencies of the vibration modes both in and out of the plane of orbit are very close and that the slight differences are attributable to the different influences of centrifugal and gravitational forces on the geometric stiffness of the string (the tether) in the two planes. More sophisticated analysis of the transverse oscillations, carried out after removing the assumption of small amplitude, has shown that the non-linearities associated mainly with the gyroscopic forces are fairly weak [12, 13].

Nonetheless, the closeness of the in-plane and out-of-plane frequencies and the coupling between the motions in the two planes that occurs when large amplitude oscillations are considered suggest the usefulness of studying the stability of the oscillations and the other non-linear phenomena which typically arise in mechanical systems characterized by internal resonance conditions $[14,15]$. In the string system the natural frequencies are all multiples of the lowest frequency, as is substantially the case in the TSS system. However, 
the modes involved in the motion are practically only those excited since the equations of motion of string do not possess the proper non-linear coupling terms $[16,17]$. On the contrary, in the TSS dynamics numerous internal resonances can occur, similar to those which can be found in cables and long cylindrical shells [18-20].

In this paper, the equations of motion obtained through Hamilton's principle are developed in a Taylor series up to the third order, so as to obtain a set of equations that can be suitably solved by approximate analytical procedures. A closed form solution has been pursued for the non-linear in-plane and out-of-plane oscillations.

An asymptotic analysis of the partial differential equations of the continuous system makes it possible to consider the modification of the frequency along with the oscillation shape with amplitude, rarely taken into account in similar studies. This is very important for the TSS in which a number of modes are involved in the phenomenon due to conditions of internal resonance. Primary and secondary instability phenomena are analyzed by using Floquet theory. The description of the regions of instability reveals a number of conditions of unstable behaviour for technical values of the TSS characteristics.

\section{THE EQUATIONS OF MOTION}

The shuttle rotates at constant angular velocity $n$ in the $X-Y$ plane along the circular orbit of radius $a$ from the centroid of the Earth (see Figure 1); in equilibrium conditions the radius and angular velocity are related by

$$
n^{2}=\mu_{e} / a^{3}
$$

where $\mu_{e}$ is the gravitational constant.

Deployment of the tethered satellite produces a variation in the orbit of the shuttle. However, since its mass is much greater than those of the satellite and of the string, this variation is neglected and in the following the distance $a$ of the shuttle from the Earth is assumed to be constant.

Two reference systems are introduced to describe the motion. The first, $O X Y Z$, is a fixed frame with its origin in the centroid of the Earth; the second, Sxyz, is connected to the shuttle and rotates at the angular velocity $n$.

The components $u(s, t), v(s, t)$ and $w(s, t)$ measure the displacements in the $x, y$ and $z$ directions respectively of the point $P(s)$ from the static straight configuration $x_{0}(s)$.

The equations of motion were obtained in reference [12] by using Hamilton's principle: no assumption for the amplitude of the displacement was introduced and the elongation $e(s, t)$ was used as a strain measure:

$$
e=\left(x^{\prime 2}+y^{\prime 2}+z^{2}\right)^{1 / 2}-1 .
$$

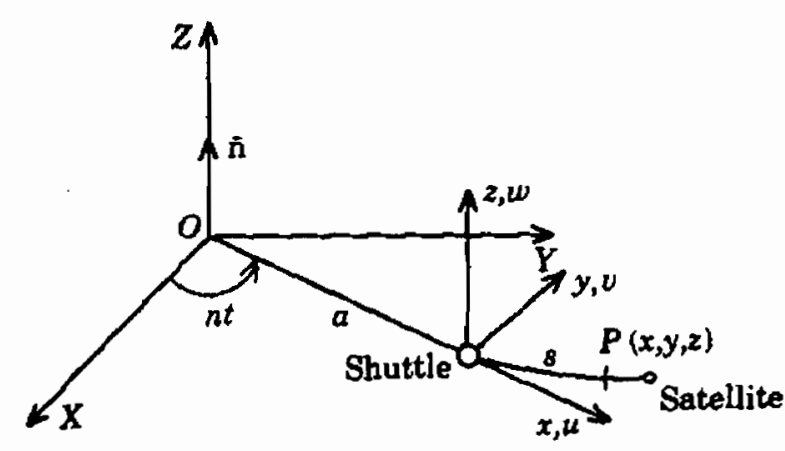

Figure 1. The tethered satellite system. 
Here the prime denotes differentiation with respect to the abscissa $s$. The equations are

$$
\begin{gathered}
\mu \ddot{u}-2 \mu n \dot{v}-\mu n^{2}\left(a+x_{0}+u\right)+\frac{\mu \mu_{e}\left(a+x_{0}+u\right)}{\left[\left(a+x_{0}+u\right)^{2}+v^{2}+w^{2}\right]^{3 / 2}} \\
-E A\left\{\left(x_{0}^{\prime}+u^{\prime}\right)\left[1-\frac{1}{\left[\left(x_{0}^{\prime}+u^{\prime}\right)^{2}+v^{\prime 2}+w^{\prime 2}\right]^{1 / 2}}\right]\right\}^{\prime}=0, \\
\mu \ddot{v}-2 \mu n \dot{u}-\mu n^{2} v+\frac{\mu \mu_{e} v}{\left[\left(a+x_{0}+u\right)^{2}+v^{2}+w^{2}\right]^{3 / 2}} \\
-E A\left\{v\left[1-\frac{1}{\left[\left(x_{0}^{\prime}+u^{\prime}\right)^{2}+v^{\prime 2}+w^{\prime 2}\right]^{1 / 2}}\right]\right\}^{\prime}=0, \\
\mu \ddot{w}+\frac{\mu \mu_{e} w}{\left[\left(a+x_{0}+u\right)^{2}+v^{2}+w^{2}\right]^{3 / 2}}-E A\left\{w^{\prime}\left[1-\frac{1}{\left[\left(x_{0}^{\prime}+u^{\prime}\right)^{2}+v^{\prime 2}+w^{\prime 2}\right]^{1 / 2}}\right]\right\}^{\prime}=0,
\end{gathered}
$$

with the boundary conditions

$$
\begin{gathered}
u(0, t)=v(0, t)=w(0, t)=0, \\
\left\{m \ddot{u}-2 m n \dot{v}-m n^{2}\left(a+x_{0}+u\right)+\frac{\mu_{e} m\left(a+x_{0}+u\right)}{\left[\left(a+x_{0}+u\right)^{2}+v^{2}+w^{2}\right]^{3 / 2}}\right. \\
\left.+E A\left(x_{0}^{\prime}+u^{\prime}\right)\left[1-\frac{1}{\left[\left(x_{0}^{\prime}+u^{\prime}\right)^{2}+v^{\prime 2}+w^{\prime 2}\right]^{1 / 2}}\right]\right\}_{s=1}=0, \\
\left\{m \ddot{v}-2 m n \dot{u}-m n^{2} v+\frac{\mu_{e} m v}{\left[\left(a+x_{0}+u\right)^{2}+v^{2}+w^{2}\right]^{3 / 2}}\right. \\
\left.+E A v^{\prime}\left[1-\frac{1}{\left[\left(x_{0}^{\prime}+u^{\prime}\right)^{2}+v^{\prime 2}+w^{\prime 2}\right]^{1 / 2}}\right]\right\}_{s=1}=0, \\
\left\{m \ddot{w}+\frac{1}{\left[\left(a+x_{0}+u\right)^{2}+v^{2}+w^{2}\right]^{3 / 2}}+E A w^{\prime}\left[1-\frac{1}{\left[\left(x_{0}^{\prime}+u^{\prime}\right)^{2}+v^{\prime 2}+w^{\prime 2}\right]^{1 / 2}}\right]\right\}_{s=1}=0 .
\end{gathered}
$$

In these equations $E A$ is the axial stiffness of the string, $l$ is the length, $\mu$ is the mass density, $m$ is the mass of the satellite and the dot denotes a derivative with respect to $t$. The physical meanings of the different terms are easily recognizable: inertial, gyroscopic, a combination of centrifugal and gravitational and elastic forces.

In order to obtain an asymptotic solution the governing equations are expanded in a Taylor series up to the third order. The analysis of the smallness of the gravitational terms makes it possible to neglect their non-linear contributions, which are very small in comparison with other elastic terms. Indeed, the ratio between gravitational and elastic non-linear terms is of $O\left[\mu n^{2} l^{3} /\left(\pi^{3} E A a\right)\right]$, which is a very small number for the actual values of the system characteristics. The same does not hold good for the linear terms; among these the gravitational force is thus included in the equations.

After introducing the dimensionless parameters

$$
\alpha^{2}=\mu n^{2} l^{2} / E A, \quad \gamma=\mu l / m, \quad \xi=l / a,
$$

and the dimensionless variables

$$
\tilde{x}=x / l, \quad \tilde{s}=s / l, \quad \tilde{t}=n t, \quad \tilde{u}=u / l, \quad \tilde{v}=v / l, \quad \tilde{w}=w / l,
$$


the governing equations at the third order, with the tilde dropped, are

$$
\begin{gathered}
\alpha^{2} \ddot{u}-2 \alpha^{2} \dot{v}-\alpha^{2} u-\frac{2 \alpha^{2} u}{\left(1+\xi x_{0}\right)^{3}}-u^{\prime \prime}-\left(\frac{v^{\prime 2}+w^{\prime 2}}{2 x_{0}^{2}}-\frac{2 u^{\prime 3}-u^{\prime} v^{\prime 2}-u^{\prime} w^{\prime 2}}{2 x_{0}^{3}}\right)^{\prime}=0, \\
\alpha^{2} \ddot{v}-2 \alpha^{2} \dot{u}-\alpha^{2} v+\frac{\alpha^{2} v}{\left(1+\xi x_{0}\right)^{3}}-\left[v^{\prime}\left(1-\frac{1}{x_{0}^{\prime}}\right)\right]^{\prime}-\left(\frac{u^{\prime} v^{\prime}}{x_{0}^{2}}-\frac{2 u^{\prime 2} v^{\prime}-v^{\prime 3}-v^{\prime} w^{\prime 2}}{2 x_{0}^{3}}\right)^{\prime}=0, \\
\alpha^{2} \ddot{w}+\frac{\alpha^{2} w}{\left(1+\xi x_{0}\right)^{3}}-\left[w^{\prime}\left(1-\frac{1}{x_{0}^{\prime}}\right)\right]^{\prime}-\left[\frac{u^{\prime} w^{\prime}}{x_{0}^{\prime 2}}-\frac{2 u^{\prime 2} w^{\prime}-v^{\prime 2} w^{\prime}-w^{\prime 3}}{2 x_{0}^{3}}\right]^{\prime}=0,
\end{gathered}
$$

with the relevant boundary conditions.

In the same way as the exact equations (3), equations (7) express that the large amplitude oscillations are coupled and all the displacement components are involved in the motion. Due to gyroscopic forces, the first two equations are also coupled in the linear terms. However, the non-linear terms are such that the motion occurs in the orbit plane if zero initial conditions on the $w$ displacement are given. The dual situation is not true, since the out-of-plane motion will always force the extensional oscillations, which in turn forces the transverse oscillation.

According to the results of the static analysis [12], the elongation $e$ can be omitted in $x_{0}^{\prime}=1+e$. If one uses

$$
f(s)=\left(1 / \alpha^{2}\right)\left(1-1 / x_{0}^{\prime}\right)
$$

and only terms of order $O\left(\xi x_{0}\right)$ are retained in the expansion of $1 /\left(1+\xi x_{0}\right)^{3}$ since $\xi \ll 1$, the equations of motion obtained are

$$
\begin{gathered}
\alpha^{2} \ddot{u}-2 \alpha^{2} \dot{v}-3 \alpha^{2} u\left(1-2 \xi x_{0}\right)-\left[u^{\prime}+\frac{v^{\prime 2}+w^{\prime 2}}{2}-\left(u^{\prime 2}-\frac{v^{\prime 2}+w^{\prime 2}}{2}\right) u^{\prime}\right]^{\prime}=0 \\
\alpha^{2} \ddot{v}+2 \alpha^{2} \dot{u}-\alpha^{2}\left[f(s) v^{\prime}\right]^{\prime}-3 \alpha^{2} \xi x_{0} v-\left[\left(u^{\prime}+\frac{v^{\prime 2}+w^{\prime 2}}{2}\right) v^{\prime}-u^{\prime 2} v^{\prime}\right]^{\prime}=0 \\
\alpha^{2} \ddot{w}+\alpha^{2} w\left(1-3 \xi x_{0}\right)-\alpha^{2}[f(s) w]^{\prime}-\left[\left(u^{\prime}+\frac{v^{\prime 2}+w^{\prime 2}}{2}\right) w^{\prime}-u^{\prime 2} w^{\prime}\right]^{\prime}=0
\end{gathered}
$$

with the boundary conditions

$$
\begin{gathered}
u(0, t)=v(0, t)=w(0, t)=0, \\
\left\{\alpha^{2} \ddot{u}-2 \alpha^{2} \dot{v}-3 \alpha^{2} u\left(1-2 \xi x_{0}\right)+\gamma\left[u^{\prime}+\frac{v^{\prime 2} w^{\prime 2}}{2}-\left(u^{\prime 2}-\frac{v^{\prime 2}+w^{\prime 2}}{2}\right) u^{\prime}\right]\right\}_{s=1}=0, \\
\left\{\alpha^{2} \ddot{v}+2 \alpha^{2} \dot{u}+\gamma \alpha^{2} f(s) v^{\prime}-3 \alpha^{2} \xi x_{0} v+\gamma\left[\left(u^{\prime}+\frac{v^{\prime 2}+w^{\prime 2}}{2}\right) v^{\prime}-u^{\prime 2} v^{\prime}\right]\right\}_{s=1}=0, \\
\left\{\alpha^{2} \ddot{w}+\gamma \alpha^{2} f(s) w^{\prime}+\alpha^{2} w\left(1-3 \xi x_{0}\right)+\gamma\left[\left(u^{\prime}+\frac{v^{\prime 2}+w^{\prime 2}}{2}\right) w^{\prime}-u^{\prime 2} w^{\prime}\right]\right\}_{s=1}=0 .
\end{gathered}
$$

This study is devoted mainly to motion with prevailing transverse components. In this context, it is possible to neglect some of the terms in the longitudinal equation. The inertia forces are negligible because the forcing terms due to the coupling with transverse oscillations depend on the transverse frequency $\omega_{t}$ which is much smaller than the lowest longitudinal frequency $\omega_{l}$; thus $\alpha^{2} \ddot{u} \simeq \alpha^{2} \omega_{l}^{2} u \ll u$. Moreover, the third term can also be 
omitted, since $3 \alpha^{2}\left(1-2 \xi x_{0}\right) \ll 1$. These assumptions make it possible to obtain an integro-differential relation among $u, v$ and $w$,

$$
u^{\prime}(s, t)=u_{1}^{\prime}-\int_{1}^{s} 2 \alpha^{2} v \mathrm{~d} s-\frac{v^{\prime 2}+w^{\prime 2}}{2}+\frac{v_{1}^{\prime 2}+w_{1}^{\prime 2}}{2}
$$

and, similarly, on the boundary

$$
u_{0}=0, \quad \gamma u_{1}^{\prime}=2 \alpha^{2} \dot{v}_{1}-\gamma\left(v_{1}^{\prime 2}+w_{1}^{\prime 2}\right) / 2 .
$$

The analysis performed is the same followed in the case of cables and beams with immovable ends, but the conciusion is quite different. In those systems the increment of axial force is constant along the axis and great in magnitude due to the stretching. Here, according to equation (11), the increment is variable and equal to the resultant of the Coriolis forces in $(s, l)$, which are small in magnitude.

By integrating equation (11) and taking account of equations (12) one obtains:

$$
u(s, t)=\frac{2 \alpha^{2}}{\gamma} i_{1} s-\int_{0}^{s} \int_{1}^{s} 2 \alpha^{2} v \mathrm{~d} s \mathrm{~d} s-\int_{0}^{s} \frac{v^{2}+w^{\prime 2}}{2} \mathrm{~d} s,
$$

which, if substituted into equations (10), makes it possible to describe the motion by only two equations in the transverse variables $v(s, t)$ and $w(s, t)$ : i.e.,

$$
\begin{gathered}
\ddot{v}-\left[f(s) v^{\prime}\right]^{\prime}-3 \xi x_{0} v-\int_{0}^{s}\left(v^{2}+w^{2}\right)^{\prime} \mathrm{d} s-\left[\frac{2}{\gamma} \dot{v}_{1} v^{\prime}-v^{\prime} \int_{1}^{s} 2 \dot{v} \mathrm{~d} s\right]^{\prime}=0, \\
\ddot{w}=[f(s) w]^{\prime}+\left(1-3 \xi x_{0}\right) w-\left[\frac{2}{\gamma} \dot{v}_{1} w^{\prime}-w^{\prime} \int_{1}^{s} 2 \dot{v} \mathrm{~d} s\right]^{\prime}=0,
\end{gathered}
$$

with the boundary conditions

$$
\begin{gathered}
v(0, t)=w(0, t)=0, \\
\ddot{v}_{1}+\gamma f(1) v_{1}^{\prime}-3 \xi x_{0}(1) v_{1}-\int_{0}^{1}\left(v^{\prime 2}+w^{\prime 2}\right)^{\cdot} \mathrm{d} s+2 \dot{v}_{1} v_{1}^{\prime}=0, \\
\ddot{w}_{1}+\gamma f(1) w_{1}^{\prime}+w_{1}\left(1-3 \xi x_{0}(1)\right)+2 v_{1} w_{1}^{\prime}=0 .
\end{gathered}
$$

Equations (14) and (15) govern the moderate amplitude transverse oscillations of the orbiting string-satellite system. The equations were derived with coherent simplifying assumptions which led to the presence of only quadratic non-linear terms descending from gyroscopic forces. These are small, which limits the importance of non-linear effects, but establishes a coupling between the equations of motion. As previously stated, the coupling is such that in-plane oscillations in the orbit plane described by only displacement component $v(s, l)$ can exist, while the out-of-plane oscillations involve both components with $w(s, t)$ prevailing.

It is useful for further analytical developments to adopt formal and more compact expressions of equations of motion and boundary conditions. These can be written as

$$
\left\{M(\bar{v})+L_{v}(v)+B(v, \dot{v})+C(w, \dot{w})\right\} \cdot \delta v=0, \quad\left\{M(\ddot{w})+L_{w}(w)+D(w, \dot{v})\right\} \cdot \delta w=0,
$$

where $M$ and $L$ are linear differential operators of $s$, and $B, C$ and $D$ are bi-linear integro-differential operators. By using for the operator $H()$ the inner product defined by

$$
H() \cdot u=\int_{D} H_{D} u \mathrm{~d} D+\int_{\Gamma} H_{r} u \mathrm{~d} \Gamma,
$$


where $H_{D}$ is the formal part of the operator in the domain $D$ and $H_{r}$ is its representation on the boundary $\Gamma$, explicit expressions for the operators which appear in equation (16) are obtained from a comparison with equations (14) and (15):

$$
\begin{aligned}
& M(\ddot{v}) \cdot \delta v=\int_{0}^{1} \ddot{v} \delta v \mathrm{~d} s+\frac{1}{\gamma} \ddot{v}_{1} \delta v_{1}, \\
& L_{v}(v) \cdot \delta v=\int_{0}^{1}\left(f v^{\prime} \delta v^{\prime}-3 \xi x_{0} \delta v\right) \mathrm{d} s-\frac{3}{\gamma} \xi x_{0}(1) \delta v_{1}, \\
& L_{w}(w) \cdot \delta w=\int_{0}^{1}\left[f w^{\prime} \delta w^{\prime}+\left(1-3 \xi x_{0}\right) w \delta w\right] \mathrm{d} s+\frac{1}{\gamma}\left(1-3 \xi x_{0}(1)\right) w_{1} \delta w_{1}, \\
& B(v, \dot{v}) \cdot \delta v=-2\left[\int_{0}^{1} \delta v \int_{0}^{s} v^{\prime} \dot{v}^{\prime} \mathrm{d} s \mathrm{~d} s+\int_{0}^{1} \delta v^{\prime} v^{\prime} \int_{1}^{s} \dot{v} \mathrm{~d} s \mathrm{~d} s\right] \\
& C(w, \dot{w}) \cdot \delta v=-2 \int_{0}^{1} \delta v \int_{0}^{s} w^{\prime} \dot{w}^{\prime} \mathrm{d} s \mathrm{~d} s-\frac{2}{\gamma} \delta v_{1} \int_{0}^{1} w^{\prime} \dot{w}^{\prime} \mathrm{d} s, \\
& D(w, \dot{v}) \cdot \delta w=-2 \int_{0}^{1} \delta w^{\prime} w^{\prime} \int_{0}^{s} \dot{d} \mathrm{~d} s \mathrm{~d} s+\frac{2}{\gamma} \dot{v}_{1} \int_{0}^{1} \delta w^{\prime} w^{\prime} \mathrm{d} s .
\end{aligned}
$$

From equations (19) it follows that

$$
B(x, y) \cdot y=0, \quad C(x, y) \cdot z=-D(x, z) \cdot y,
$$

which will be used in the following.

\subsection{SMALI, AMPLITUDE OSCILLATIONS}

The linearized equations of motion, obtained from equations (14) with the non-linear terms omitted, are uncoupled: the first describes the small amplitude oscillations in the plane of orbit and the second those out of plane. These are differential equations with variable coefficients, but $f(s)$ is very weakly dependent on $s$ and can be represented by a suitable constant value $f_{0}[9]$. Under these assumptions the analytical solution is, in-plane,

$$
v(s, t)=A_{p} \sin p s \sin \omega_{v} t, \quad \operatorname{tg} p=\gamma / p, \quad \omega_{v}^{2}=p^{2} f_{0},
$$

and, out-of plane,

$$
w(s, t)=A_{q} \sin q s \sin \omega_{k} t, \quad \operatorname{tg} q=\gamma / q, \quad \omega_{k}^{2}=q^{2} f_{0}+1 .
$$

The only difference is in the frequencies and is due to the slightly different contributions of the gravitational and centrifugal forces to the stiffnesses in the two planes. These differences strongly decrease with the mode number; indeed, for increasing values of $p$ and $q$, equations $\left(2 l_{3}\right)$ and $\left(22_{3}\right)$ give $\omega_{1} \simeq \omega_{w}$.

In the section on numerical investigations, reference will be made to two tethered satellite systems that differ only in the lengths of the strings. The following geometric and mechanical parameters, which cover a technical field of interest, were considered: $n^{2}=1.35 \times 10^{-6} \mathrm{~s}^{-2}, a=\left(\mu_{e} / n^{2}\right)^{1 / 3}=6657 \mathrm{~km}, m=500 \mathrm{~kg}, l=5-100 \mathrm{~km}, m / A=1.44 \times$ $10^{3} \mathrm{~kg} / \mathrm{m}^{3}, A=4 \times 10^{-6} \mathrm{~m}^{2}, E=7 \times 10^{10} \mathrm{~N} / \mathrm{m}^{2}$.

The frequencies of the first five transverse modes are shown in Table 1 . The first frequencies of the two systems are very similar and quite distant from the others, since the first mode is a pendulum-type mode while the others involve string deformation. In 


\section{TABLE 1}

Linear frequencies of transverse modes

\begin{tabular}{lcccccc}
\hline & & First mode & Second mode & Third mode & Fourth mode & Fifth mode \\
\hline$l=5 \mathrm{~km}$ & $\omega_{\mathrm{r}}$ & 1.736 & 23.022 & 45.845 & 68.712 & 91.590 \\
& $\omega_{i r}$ & 2.004 & 23.044 & 45.856 & 68.719 & 91.595 \\
& $p=q$ & 0.238 & 3.160 & 6.292 & 9.431 & 12.571 \\
$l=100 \mathrm{~km}$ & $\omega_{v}$ & 1.725 & 6.577 & 12.265 & 18.122 & 24.030 \\
& $\omega_{*}$ & 1.995 & 6.652 & 12.306 & 18.149 & 24.051 \\
& $p=q$ & 0.909 & 3.464 & 6.461 & 9.545 & 12.658 \\
\hline
\end{tabular}

particular, the set of frequencies, apart from the first one, practically follows the law $p=q \cong(k-1) \pi$, which is the same spectrum as that of the taut string.

\section{MONOFREQUENT OSCILLATIONS}

This section is concerned with the solution of equations (16) for only the cases of monofrequent oscillations where all the points of the system exhibit periodic motion [22]. In linear dynamics, when a system takes an eigenfunction as its initial shape, the motion of each point is synchronous with the same frequency and, in general, the spatial shape does not change during motion. In non-linear dynamics, monofrequent oscillations have similar properties: i.e., each point of the system moves with the same frequency or its multiple, but the frequency of oscillation varies with the amplitude, as in a non-linear single degree of freedom system; moreover, the shape of the monofrequent oscillation also varies with the amplitude.

The present study is limited to those monofrequent oscillations for which, as the amplitude tends toward zero, the shape tends toward an eigenfunction. The deformed configuration is mostly described by an eigenfunction and its modification with the amplitude is represented by the contribution of other modes involved by non-linear coupling.

The analytical solution can be pursued by either asymptotic or harmonic balance methods. The evolution of the phenomenon can be more easily understood if a perturbation approach is followed. The linear mode close to the monofrequent oscillation under consideration is assumed to be the generating solution and the analysis of non-linear terms makes it possible to predict the modes forced in the solution at higher order. For the tethered satellite system when an in-plane mode with frequency $\omega_{k}$ is assumed as the generating solution, the motion is governed only by equation $\left(16_{1}\right)$. At higher order the non-linear term $B(v, \dot{v})$, in which $v$ is the generating solution, forces other modes, of which the only important one is that with frequency $\omega_{j}$ close to $2 \omega_{k}$. Similarly, when an out-of-plane mode with frequency $\omega_{k}$ is assumed as the generating solution of equation $\left(16_{2}\right)$, the term $C(w, w)$ in equation $\left(16_{1}\right)$ forces the occurrence of in-plane modes, of which the only one to be retained has a frequency $\omega_{j}$ close to $2 \omega_{k}$.

For these systems the condition of internal resonance $\left(\omega_{1} \simeq 2 \omega_{k}\right)$ is frequently found, as can be argued from Table 1 . Since the perturbation technique could fail because the higher order components tend to infinity, when the periodic solutions only are sought it is preferable to use the harmonic balance method which is valid near or far from the internal resonance. However, according to the previous considerations, the periodic solution is studied by taking only two components, the $k$ th mode and the $j$ th mode with $\omega$, close to $2 \omega_{k}$. 


\section{1. oscillations IN THE PLANE OF THE ORBIT}

This section is concerned with the monofrequent in-plane oscillations of non-linear frequency $\Omega_{k}$. A periodic solution with only two components vibrating with frequency $\Omega_{k}$ and $2 \Omega_{\star}$ is sufficient to describe the phenomenon accurately:

$$
v(s, t)=A_{k} \varphi_{k}(s) \cos \Omega_{k} t+A_{j} \varphi_{j}(s) \sin 2 \Omega_{k} t .
$$

Here $\varphi_{k}$ and $\varphi_{j}$ are the linear modes with frequency $\omega_{k}$, close to $\Omega_{k}$ for small amplitude, and $\omega_{j}$, which differs from $2 \omega_{k}$ by a small quantity $\sigma$ :

$$
\omega_{j}+\sigma=2 \omega_{k} \text {. }
$$

In equation (23), $A_{k}$ and $A_{j}$ are the amplitudes of the two modes, suitably normalized. The relation (23) and its derivative is substituted in equation $\left(16_{1}\right)$ along with $\delta v(s, t)$ again expressed by means of equation (23). By performing the integrals according to equations (18) and (19) and separating terms in $\cos \Omega_{k} t \delta A_{k}$ and $\sin 2 \Omega_{k} t \delta A_{j}$, the following two equations are obtained:

$$
m_{k} A_{k}\left(\omega_{k}^{2}-\Omega_{k}^{2}\right)+\Omega_{k} b_{k k j} A_{k} A_{j}=0, \quad m_{j} A_{j}\left(\omega_{j}^{2}-4 \Omega_{k}^{2}\right)-\frac{1}{2} \Omega_{k} b_{j k k} A_{k}^{2}=0 .
$$

Here $m_{k}, m_{j}$ and $b_{r s t}$ are given in Appendix A.

The first equation gives the frequency-amplitude relationship,

$$
\Omega_{k}=\omega_{k}\left[1+\left(b_{k k j} / \omega_{k} m_{k}\right) A_{j}\right]^{1 / 2}+O\left(A_{j}^{2}\right),
$$

and the second, together with equation (26), makes it possible to obtain a relationship between $A_{j}$ and $A_{k}$,

$$
\left(b_{k k j} / m_{k}\right) A_{j}^{2}+\sigma A_{j}+\left(b_{j k k} / 8 m_{j}\right) A_{k}^{2}=0,
$$

which governs the modification of the shape with oscillation amplitude.

\subsection{OSCILLATIONS OUT OF THE PLANE OF THE ORBIT}

On account of the nature of the coupling terms, the monofrequent oscillation near the $k$ th linear out-of-plane mode is characterized by a component that is also in the orbit plane. Thus, the periodic solutions are described by both transverse displacements, the prevailing displacement $w(s, t)$ oscillating in the linear $k$ th eigenfunction $\psi_{k}(s)$ and with frequency $\Omega_{k}$ near $\omega_{k}$ and the forced displacements $v(s, t)$ oscillating in the linear $j$ th eigenfunction $\varphi_{j}(s)$ and with frequency $2 \Omega_{k}$ close to $\omega_{j}$ :

$$
v(s, t)=A_{j} \varphi_{j} \sin 2 \Omega_{k} t, \quad w(s, t)=A_{k} \psi_{k} \cos \Omega_{k} t
$$

If equations (28) are introduced into equations (16) and the same procedure outlined in the study of in-plane monofrequent oscillations is followed, a similar system of two equations is obtained,

$$
m_{j} A_{j}\left(\omega_{j}^{2}-4 \Omega_{k}^{2}\right)-\frac{1}{2} \Omega_{k} c_{j k k} A_{k}^{2}=0, \quad m_{k} A_{k}\left(\omega_{k}^{2}-\Omega_{k}^{2}\right)+\Omega_{k} d_{k k j} A_{k} A_{j}=0,
$$

the solution of which gives the frequency-amplitude relationship and the relationship between the amplitudes of the two modes involved in the motion:

$$
\Omega_{k}=\omega_{k}\left[1+\left(d_{k k j} / \omega_{k} m_{k}\right) A_{j}\right]^{1 / 2}+O\left(A_{j}^{2}\right), \quad\left(d_{k k j} / m_{k}\right) A_{i}^{2}+\sigma A_{j}+\left(c_{j k k} / 8 m_{j}\right) A_{k}^{2}=0 .
$$

The coefficients $c_{r s t}$ and $d_{r s}$ are given in Appendix A. 


\subsection{COMMENTS}

The peculiar features of monofrequent oscillations emerge from equations (27) or (31) which establish a relation between the two amplitudes. This means that the deformed shape of the system changes with amplitude and, moreover, according to equations (23) or (28), does not remain constant during oscillation, as it does in the case of linearized motion. Nevertheless, it repeats itself every period.

In both cases studied, the in-plane and out-of-plane oscillations, the frequency correction is due to the emergence of a new component $A_{j}$, which produces a modification of the fundamental mode shape. If this modification is not taken into account, the frequency $\Omega_{k}$ is the same as the linear frequency.

When the condition of internal resonance occurs, $\sigma=o(1), A_{j}=O\left(A_{k}\right)$ is obtained from equation (27) or equation (31) apart from a range of $A_{k}$ close to the origin. This means that the reference $k$ th mode no longer prevails beyond this range, but the motion in a condition of internal resonance is described by both the resonant modes. When it is out of resonance, $\sigma=O(1), A_{j}=O\left(A_{k}^{2}\right)$ in a range of not too large amplitude, and the oscillation is mainly described by the $k$ th mode with a small contribution by the $j$ th mode.

The features outlined above are illustrated in Figure 2, in which two cases with different $\sigma$ are considered. In each case two curves (heavy line) of monofrequent oscillations are found, one (a) emanating from the origin, the other (b) from a value $A_{j} \neq 0$. Curve (a) is an oscillation in which the amplitude of the fundamental mode $A_{k}$ always prevails on the forced mode $A_{j}$ and when the amplitude $A_{k}$ of the governing mode tends to zero $A_{j}$ also tends to zero: this is the linear solution. Curve (b) starts from a bifurcation point $A_{j}^{c}$ on the path $A_{k}=0, A_{j} \neq 0$ which is the solution of systems (25) or (29) with only one component and represents monofrequent in-plane oscillations described by the $j$ th mode. They are stable for $0<A_{j}<A_{j}^{c}$, while they are unstable for $A_{j} \geqslant A_{c}^{j}$. This curve (b) thus represents the bifurcated solution path for which the $k$ th mode does not always prevail and the solution does not tend to the linear one as $A_{k}$ tends to zero. The curves (a) and (b) degenerate into the dotted lines emanating from the origin, when perfect resonance occurs $(\sigma=0)$. It is useful to note that the monofrequent oscillations characterized by two components, curves (a) and (b), are always stable for small variations of $A_{k}$ and/or $A_{j}$ [21].

In Figure 2 curves (c) are the non-resonant solution obtained by the Lindstedt-Poincaré perturbation method which leads to a relation between $A_{j}$ and $A_{k}$ coincident with equations (27) or (31) where the terms in $A_{j}^{2}$ are neglected. It well describes the phenomenon for a large range of $A_{k}$ when $\sigma=O(1)$, while in conditions of internal resonance, $\sigma=o(1)$, this solution soon diverges from the harmonic balance solution. Thus, when the solution can be suitably determined by the perturbation method, this may be preferable to the harmonic
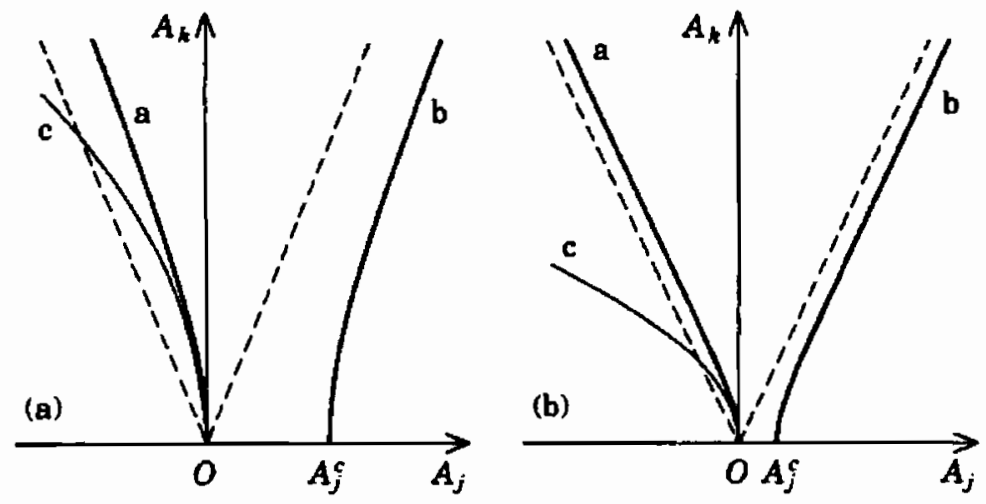

Figure 2. The relation between the amplitudes of the two components of monofrequent oscillations. (a) $\sigma=O(1),($ b) $\sigma=O(1)$ 
balance method, since the significant modes involved in the solution should not be selected a priori but are straightforwardly determined during the solution procedure.

\section{STABILITY ANALYSIS}

A disturbance $\eta(s, t), \zeta(s, t)$ is superimposed on the steady oscillation $v_{s}(s, t), w_{s}(s, t)$,

$$
v(s, t)=v_{s}(s, t)+\eta(s, t), \quad w(s, t)=w_{s}(s, t)+\zeta(s, t),
$$

where each component of the disturbance is described by one suitably selected mode,

$$
\eta(s, t)=\varphi_{t}(s) \eta(t), \quad \zeta(s, t)=\psi_{l}(s) \zeta(t) .
$$

Equations (32) are introduced into equations (16); linearizing with respect to $\eta(t)$ and $\zeta(t)$ leads to

$$
\begin{gathered}
{\left[M(\ddot{\eta})+L_{v}(\eta)+B\left(v_{s}, \dot{\eta}\right)+B\left(\eta, \dot{v}_{s}\right)+C\left(w_{s}, \zeta\right)+C\left(\zeta, \dot{w}_{s}\right)\right] \cdot \delta \eta=0,} \\
{\left[M(\zeta)+L_{w}(\zeta)+D\left(w_{s}, \dot{\eta}\right)+D\left(\zeta, \dot{v}_{s}\right)\right] \cdot \delta \zeta=0 .}
\end{gathered}
$$

If equations (33) are used and the Galerkin technique applied, one has

$$
\left[\begin{array}{cc}
m_{l} & 0 \\
0 & m_{l}
\end{array}\right]\left\{\begin{array}{l}
\ddot{\eta} \\
\xi
\end{array}\right\}+\left(\left[\begin{array}{cc}
k_{i} & 0 \\
0 & k_{l}
\end{array}\right]+\left[\begin{array}{cc}
f_{11} & f_{12} \\
0 & f_{22}
\end{array}\right]\right)\left\{\begin{array}{l}
\eta \\
\zeta
\end{array}\right\}+\left[\begin{array}{cc}
0 & g_{12} \\
-g_{12} & 0
\end{array}\right]\left\{\begin{array}{l}
\dot{\eta} \\
\zeta
\end{array}\right\}=\left\{\begin{array}{l}
0 \\
0
\end{array}\right\}
$$

where the quantities $m_{h}, k_{h}, f_{r s}$, and $g_{r s}$ are as defined in Appendix B.

\subsection{STABILITY OF THE OSCILLATIONS IN THE ORBIT PLANE}

The stability of the oscillations in the orbit plane for both out-of-plane and in-plane perturbations is considered in this section. The analysis is governed in turn by only one of the two equations (35); indeed, when the in-plane motion is considered (equation (23)), equations (35) are uncoupled, since $f_{12}$ and $g_{12}$ vanish. The stability for an out-of-plane disturbance $\zeta(s, t)$ is then governed by equation $\left(35_{2}\right)$ :

$$
m_{1} \ddot{\zeta}(t)+\left(k_{1}+f_{2 z}\right) \zeta(t)=0 .
$$

Upon using equation (23) to calculate $f_{22}$ and introducing a new time variable $\tau=\left(\Omega_{k} / 2\right) t+(\pi / 4)$, equation (36) becomes

$$
\zeta(t)+\left(\delta^{2}+\epsilon_{1} \cos 2 \tau+\epsilon_{2} \cos 4 \tau\right) \zeta(t)=0,
$$

where

$$
\delta=2 \omega_{l} / \Omega_{k}, \quad \epsilon_{1}=4\left(A_{k} / m_{l} \Omega_{k}\right) d_{l k k}, \quad \epsilon_{2}=-8\left(A_{j} / m_{1} \Omega_{k}\right) d_{l j} .
$$

The differential equation (37) has periodic coefficients of frequency 2; according to Floquet theory, the boundaries of the first instability region are associated with the periodic solutions of frequency 1 . The function $\zeta(\tau)$ is expanded in series of $\sin \tau$ and $\cos \tau$,

$$
\zeta(\tau)=a_{1} \cos \tau+b_{1} \sin \tau+a_{3} \cos 3 \tau+b_{3} \sin 3 \tau+\cdots,
$$

and is substituted into equation (37). The vanishing of the terms in $\sin \tau, \sin 3 \tau, \cos \tau$ and $\cos 3 \tau$ leads separately to two uncoupled eigenvalue problems which give the boundaries of the unstable regions as functions of $\delta, \epsilon_{1}$ and $\epsilon_{2}$ :

$$
\left(\delta^{2}-1 \pm \epsilon_{1} / 2\right)\left(\delta^{2}-9\right)-\left[\left(\epsilon_{1}+\epsilon_{2}\right) / 2\right]^{2}=0 .
$$

Recalling equations (38) and retaining terms up to $A_{k}^{2}$ leads to

$$
\delta^{2}=1 \pm\left(\epsilon_{1} / 2\right)-\left[\left(\epsilon_{1} \pm \epsilon_{2}\right) / 32\right]^{2}+\cdots
$$


A primary instability region emanates from $\Omega_{k}=2 \omega_{l}$. This circumstance can be easily verified between two modes higher than the first one (see Table 1). As might be expected, the instability region is very narrow, on account of the weak non-linearities of the system, but the phenomenon is, however, worthy of note, since the ratio between an in-plane and an out-of-plane frequency is very close to 2.

To determine the region of secondary instability, the function $\zeta(\tau)$ is expanded in series in $\sin 2 \tau$ and $\cos 2 \tau$ plus a constant term:

$$
\zeta(\tau)=a_{0}+a_{2} \cos 2 \tau+b_{2} \sin 2 \tau+a_{4} \cos 4 \tau+b_{4} \sin 4 \tau+\cdots .
$$

Substituting equation (42) into equation (37), and zeroing the terms with the same harmonics leads to the equations describing the boundaries of the unstable region,

$$
\delta^{2}=4-\left(\epsilon^{2} / 2\right)+\frac{5}{48} \epsilon_{1}^{2}, \quad \delta^{2}=4+\left(\epsilon_{2} / 2\right)-\frac{1}{48} \epsilon_{1}^{2},
$$

which states that the second unstable region emanates from $\Omega_{k}=\omega_{l}$.

The stability for an in-plane disturbance $\eta(s, t)$ is governed by equation $\left(35_{1}\right)$,

$$
m_{i} \ddot{\eta}(t)+\left(k_{i}+f_{11}\right) \eta(t)=0,
$$

and as in the previous case equation (44) is transformed into

$$
\ddot{\eta}(t)+\left(\delta^{2}+\epsilon_{1} \cos 2 \tau+\epsilon_{2} \cos 4 \tau\right) \eta(t)=0,
$$

where

$$
\delta=2 \omega_{i} / \Omega_{k}, \quad \epsilon_{1}=4\left(A_{k} / m_{l} \Omega_{k}\right) b_{i i k}, \quad \epsilon_{2}=-8\left(A_{j} / m_{l} \Omega_{k}\right) b_{i j} .
$$

The instability region emanates from $\Omega_{k}=2 \omega_{i}$, a circumstance which can effectively occur since for each in-plane mode there is always a higher mode with almost double frequency. The boundaries of the region are again described by equations (41) with the symbols therein as given by equations (46).

For the in-plane disturbance, there is no point in considering the case of secondary instability. The disturbance would coincide with the steady mode itself, a condition-free oscillations of a conservative single-degree-of-freedom system-in which the motion is naturally stable.

\subsection{STABILITY OF THE OSCILLATIONS OUT OF THE ORBIT PLANE}

The steady oscillation has two components of different order, described by equations (28) and (31). In this case the system (35) is effectively coupled and a spatial disturbance $\eta(s, t), \zeta(s, t)$ must be considered. By referring to a new time variable $\tau=\Omega_{k} t+\pi / 2$, a non-dimensionalized form of equations (35) is obtained,

$$
\begin{array}{r}
\ddot{\eta}(t)+\left[\delta^{2}+\epsilon_{1} \cos 2 \tau\right] \eta(t)+\epsilon_{3} \delta[\zeta(t) \sin \tau]^{*}=0, \\
\zeta(t)+\left[v^{2} \delta^{2}+\epsilon_{2} \cos 2 \tau\right] \zeta(t)-\epsilon_{4} \delta \dot{\eta}(t) \sin \tau=0,
\end{array}
$$

where

$$
\begin{gathered}
\delta=\omega_{i} / \Omega_{k}, \quad v=\omega_{1} / \omega_{i}, \\
\epsilon_{1}=\left(2 / m_{i} \Omega_{k}\right) A_{j} b_{i j}, \quad \epsilon_{2}=-\left(2 / m_{l} \Omega_{k}\right) A_{j} d_{l j}, \quad \epsilon_{3}=\left(A_{k} / m_{i} \omega_{i}\right) c_{i k l}, \quad \epsilon_{4}=\left(m_{i} / m_{l}\right) \epsilon_{3} .
\end{gathered}
$$

System (47) has periodic coefficients of frequency 1 . The boundaries of the primary instability regions are obtained by the periodic solution $\eta(t), \zeta(t)$ with frequency $1 / 2$. The analysis furnishes two coincident curves with which no instability region is associated. This 
occurs precisely because more components than the two resonant modes are involved in the motion. Thus the accuracy of the solution would depend strongly on the choice of the modes involved.

From a numerical point of view, the coefficient $c_{i k t}$ could be evaluated for different choices of $l$ th mode and the maximum value selected. However, since the secondary instability region concerns the internal resonance $\omega_{k}^{*} \simeq \omega_{i}^{v}$, the analysis of the non-linear terms in equations (16) could facilitate this selection. Indeed the resonant terms come from the modes oscillating with frequency $2 \Omega_{k}$ forced by the prevailing non-linear term $C(w, \dot{w})$. All the modes are forced but the most important one is just the $l$ th mode with its frequency $\omega_{1} \simeq 2 \omega_{k}$. In this way the $l$ th mode must be selected in equations (33) to be used in equations (47). The validity of this will be shown in the numerical applications in the next section.

\section{NUMERICAL INVESTIGATION}

The phenomenon of non-linear coupling on which the stability of motion depends mainly affects modes higher than the first, since this latter mode is a nearly rigid body mode. The study will also include consideration of the first modes, but will start from the second.

\subsection{N-PLANE OSCILLATIONS}

The first monofrequent oscillation considered in the orbit plane is close to the second mode. According to the results obtained in section 3.1, the motion is described by a prevailing component of the second mode, with amplitude dependent frequency $\Omega_{2} \simeq \omega_{2}^{p}$ and shape $\varphi_{2}(s)$, and another component of the third mode, with frequency $2 \Omega_{2} \approx \omega_{3}^{\varepsilon}$ and shape $\varphi_{3}(s)$, the contributions of which are measured by the amplitudes $A_{2}$ and $A_{3}$ respectively. For the two cases in which the length of the string is 5 and $100 \mathrm{~km}$, the relationship between $A_{2}$ and $A_{3}$ for the stationary solution, described by equation (27), is shown in Figure 4(a).

With attention limited to the solutions for which $A_{3}$ tends to zero when $A_{2}$ tends to zero (curve a in Figure 2) it follows from Figure 4(a) that $A_{3}$ is smaller than $A_{2}$ but its presence is responsible for the frequency modification, which is important in the stability analysis, as will be shown.

Since there is no out-of-plane mode close to the region of primary instability, $\omega_{1} \simeq \omega_{k} / 2$, the instability of the second mode oscillation can only be produced by a phenomenon of

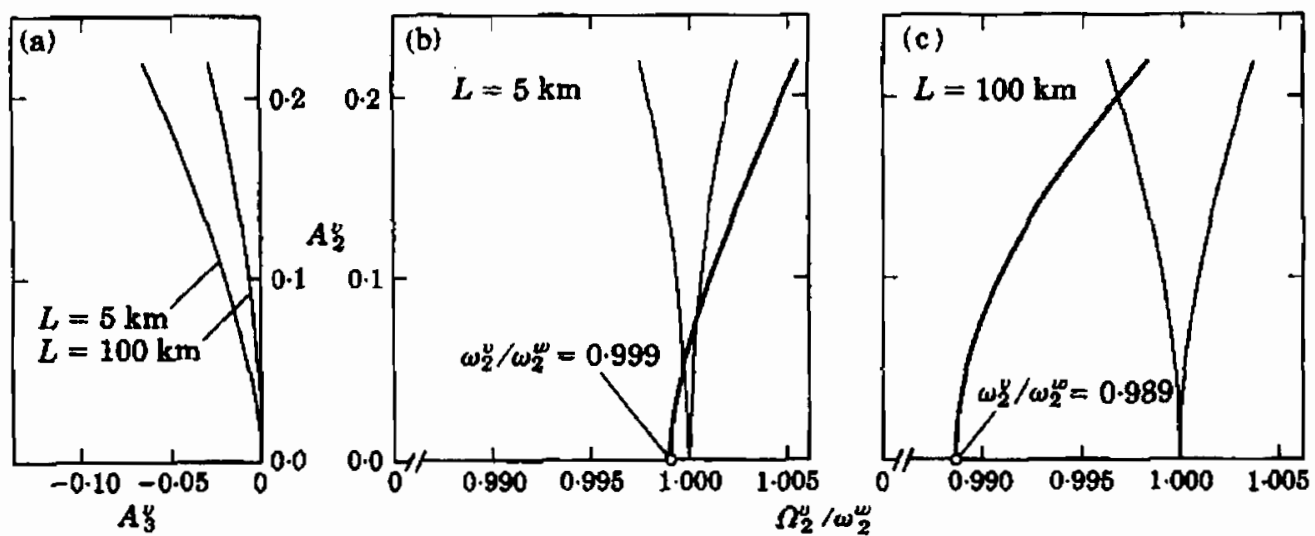

Figure 4. Monofrequent in-plane oscillations close to the second mode: the amplitudes of the two modes involved and the secondary instability regions for two string lengths under out-of-plane disturbance. 


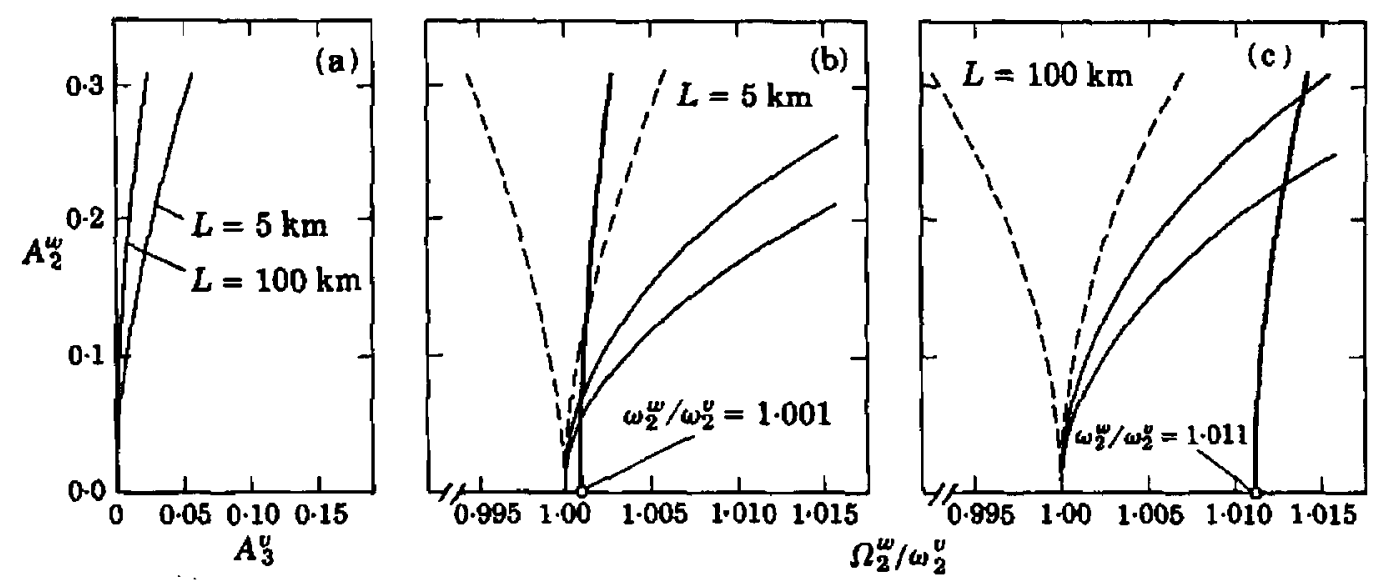

Figure 6. Monofrequent out-of-plane oscillations close to the second mode: the amplitudes of the two modes involved and the secondary instability regions for two string lengths under in-plane disturbance.

These regions have been obtained by considering a disturbance described by the resonant in-plane mode $\left(i=2, \omega_{1} \simeq \omega_{k}\right)$ and the third out-of-plane mode with a double frequency $\left(l=3, \omega_{l} \approx 2 \omega_{k}\right)$, which is the correct choice according to the considerations developed in section 4.2. If the apparently more straightforward choice has been followed and the two resonant modes, $i=2$ and $l=2$, had been assumed as a disturbance the dotted boundaries of instability would be found, which does not accurately describe the actual phenomenon. Indeed, in the truncated description of the problem the phenomenon of internal resonance $\left(\omega_{2}^{v} \simeq \omega_{2}^{*}\right)$ responsible for unstable behaviour requires one to take into account the third out-of-plane mode $\left(\omega_{3}^{w} \simeq 2 \omega_{2}^{*}\right)$, which is the most important among those involved in the motion. Its omission strongly modifies the instability region.

\section{CONCLUSIONS}

After condensing the longitudinal displacement, the non-linear transverse oscillations of a tethered satellite system can be adequately described by two equations. These reveal weak quadratic non-linear terms, due mainly to gyroscopic forces. By using the harmonic balance method, the monofrequent oscillations have been determined.

The modification of frequency with the amplitude, which would be negligible in a weakly non-linear system, is here appreciable because, it is always associated with a modification in the shape of the oscillation, due to an internal resonance phenomenon which characterizes this orbiting system. The oscillation shape is in fact described by the fundamental mode with the linear frequency close to the harmonic considered and by a contribution of other modes, of which the double frequency mode is the most important to retain. Since this contribution increases with the amplitude, the shape also changes with the amplitude. Moreover, although the motion is periodic, the shape changes during each period on account of the presence of higher harmonics, but is the same at the end of the period. These shapes, the role of which is similar to that of linearized modes, could merit the name of non-linear modes, used elsewhere to denote the harmonic motion of multi-degree-of-freedom systems $[15,23]$ and recently re-analyzed in a more general context $[24,25]$.

Primary and secondary stability of monofrequent oscillations in and out of the plane of orbit were studied through an asymptotic solution of two variational equations obtained by assuming one mode to describe each disturbance in the two planes. While in the study of primary instability it is clear which two modes are involved in the resonance 
phenomenon, in the case of secondary instability more than two modes are involved and an accurate selection of modes needs to be made to describe the disturbance.

The stability analysis performed for different geometrical characteristics of the TSS reveals unstable behaviour in both monofrequent oscillations for certain values of the amplitude, although the non-linearities of the system are very weak. Again, this occurs because the condition for numerous internal resonances is almost satisfied.

\section{ACKNOWLEDGMENT}

This research was partially supported by Ministry of University and Scientific Technological Research funds during 1991.

\section{REFERENCES}

1. D. D. LANG and R. K. Noltng 1967 Gemini Summary Conference, Houston, Texas, NASA SP-138. Operations with tethered space vehicles.

2. G. Colombo, E. M. Gaposchkin, M. D. Grassi and G. C. Weiffenbach 1975 Meccanica 10, 3-20. A shuttle borne tool for low-orbital-altitude research.

3. V. J. Mod1, G. Chang-Fu, A. K. MISRA and D. M. XU 1982 Acta Astronautica 9(6-7), 437-443. On the control of the space shuttle based tethered systems.

4. V. V. BeLETSKII and E. M. LEVIN 1985 Acta Astronautica 12(S), 285-291. Dynamics of the orbital cable system.

5. A. D. MISRA and V. J. MODI 1986 Proceedings of the NASA/AlAA/PSN International Conference on Tethers in Space, 667-719. A survey on the dynamics and control of tethered satellite systems.

6. S. Bergamaschi, S. Cusinato and A. Sinopol 1986 Proceedings of the AlAA 24th Aerospace Sciences Meeting. New York: American Institute of Aeronautics and Astronautics. A continuous model for tether elastic vibrations in TSS.

7. A. H. VON FLoTow 1988 Journal of Guidance, Control, and Dynamics 11(4), 357-364. Some approximations for the dynamics of tethered space-craft.

8. A. H. VON Flotow and N. M. WERELY 1990. Mechanics and Control of Large Flexible Structures (J. L. Junkins, editor), Progress in Astronautics and Aeronautics 129, 667-696. Insights and approximations in dynamic analysis of spacecraft tethers.

9. M. Pasca, M. Pignataro and A. Luonco 1990 Journal of Guidance, Control, and Dynamics 14(2), 312-320. Three-dimensional vibrations of tethered satellite systems.

10. X. HE and J. D. POWELL 1990 Journal of Guidance, Control, and Dynamics 13(1), 104-112. Tether damping in space.

11. G. DE MATTES and L. M. DE Socro 1991 Journal of Guidance, Control, and Dynamics 14(6), 1129-1135. Dynamics of a tethered satellite subjected to aerodynamics forces.

12. M. Pasca, A. Luongo, M. Pignataro and F. Vestroni 1987 Department of Structural and Geotechnical Engineering, University of Rome, Italy, Report (3). Free dynamics of the shuttle - tethered-satellite system.

13. F. VESTRONI and A. L. LuONGo 1991 in Trends in Applications of Mathematics to Mechanics (W. SCHNEIDER, H. TROGER, F. ZIEGLeR, edjtors). London: Longman. Perturbation analysis of finite oscillations of an orbiting string.

14. A. H. NAYFEH and D. T. MOOK 1979 Nonlinear Oscillations. New York: John Wiley.

15. W. SzempLInSKa-StupNicka 1990 The Behowiour of Nonlinear Vibrating Systems, Volumes I \& II, Dordrecht, The Netherlands: Kluwer Academic.

16. J. W. MiLes 1984 Journal of the Acoustical Society of America 75, 1505-1510. Resonant nonplanar motion of a stretched string.

17. A. K. BNA and J. M. JOHNSON 1992 Philosophical Transactions of the Royal Society of London 338, $1-41$. On the amplitude dynamics and crisis in resonant motion of stretched strings.

18. C. L. LEe and N. C. PERKJNs 1992 ASME Winter Annual Meeting. Three-dimensional oscillations of suspended cables involving simultaneous internal resonances.

19. A. H. Nayfeh and B. Balachandran 1989 Applied Mechanics Review 42(11), 175-202. Modal interactions in dynamical and structural systems. 
20. R. A. RaOuf and A. H. NAYFEH 1990 Computers and Structures 35, 163-173. One-to-one autoparametric resonances in infinitely long cylindrical shells.

21. A. LuONGo and F. VESTRONI 1990 Proceedings of the 10th Theoretical and Applied Mechanics Italian Conference, Pisa, Ilaly. Nonlinear oscillations of an orbiting string (in Italian).

22. N. Bogolioubov and I. Mrtropolski 1962 Les Méthodes asymptotiques en théorie des oscillations non linéaires. Paris: Gautier-Villars Editeur.

23. R. M. ROSENBERG 1966 Advances in Applied Mechanics 9, 155-242. On non-linear vibrations of systems with many degrees-of-freedom.

24. A. Vakakis 1990 Ph.D. Thesis, California Institute of Technology. Analysis and identification of linear and non-linear normal modes in vibrating systems.

25. S. W. Shaw and C. Pierre 1993 Journal of Sound and Vibration 164, 85-124. Normal modes for non-linear vibratory systems.

\section{APPENDIX A}

The following positions are introduced:

$$
\begin{gathered}
m_{k}=\varphi_{k} \cdot M\left(\varphi_{k}\right), \quad m_{j}=\psi_{j} \cdot M\left(\psi_{j}\right), \\
b_{i j k}=\varphi_{i} \cdot B\left(\varphi_{j}, \varphi_{k}\right), \quad c_{i j k}=\varphi_{i} \cdot C\left(\psi_{j}, \psi_{k}\right), \quad d_{i j k}=\psi_{i} \cdot D\left(\psi_{j}, \varphi_{k}\right) .
\end{gathered}
$$

The operators $B, C, D$ and $M$ have been defined in equations (18) and (19).

\section{APPENDIX B}

The quantities introduced in equation (35) are defined as follows:

$$
\begin{array}{ccc}
m_{1}=\varphi_{i} \cdot M\left(\varphi_{i}\right), \quad m_{l}=\psi_{i} \cdot M\left(\psi_{l}\right), \quad k_{i}=\varphi_{i} \cdot L_{v}\left(\varphi_{i}\right), & k_{l}=\psi_{l} \cdot L_{w}\left(\psi_{l}\right), \\
g_{11}=\varphi_{i} \cdot B\left(v_{s}, \varphi_{i}\right), & g_{12}=\varphi_{1} \cdot C\left(w_{s}, \psi_{l}\right), & g_{21}=\psi_{l} \cdot D\left(w_{s}, \varphi_{l}\right), \\
f_{11}=\varphi_{i} \cdot B\left(\varphi_{l}, \dot{v}_{s}\right), & f_{12}=\varphi_{i} \cdot C\left(\psi_{l}, \dot{w}_{s}\right), & f_{22}=\psi_{l} \cdot D\left(\psi_{l}, \dot{v}_{s}\right) .
\end{array}
$$

Due to the properties (20), $g_{11}=0$ and $g_{21}=-\dot{g}_{12}$. Due to the symmetry of $C, f_{12}=\dot{g}_{12}$. 